\title{
TELECOMUNICACIONES COMO FACTOR DE INTEGRACIÓN LABORAL: EL TRABAJO FEMENINO EN LA RED TELEFÓNICA INSULAR DE TENERIFE
}

\author{
Rafael Pérez Jiménez* \\ Francisco Quintana Navarro** \\ Universidad de Las Palmas de Gran Canaria
}

\section{RESUMEN}

En este trabajo se estudia el proceso de integración de la mujer en las primeras empresas de telecomunicación en Canarias en el periodo anterior a la Guerra Civil. Para ello se usa, como ejemplo de evolución, la de la Red Telefónica Insular de Tenerife, que fue paulatinamente evolucionando hasta un modelo de integración avanzado para la época. Se describen las distintas etapas de ese proceso y se establece una comparación con la situación del Cuerpo de Telégrafos y de la Compañía Telefónica.

Palabras clave: Tenerife, Red Insular de Teléfonos, telefonistas, género, integración.

\section{TELECOMMUNICATIONS AS A LABOR INTEGRATION FACTOR: FEMALE WORK IN THE TENERIFE INSULAR TELEPHONE NETWORK}

\section{Abstract}

In this work the process of integration of women in the first telecommunication companies in the Canary Islands in the period before the Civil War is considered. We use, as an example of evolution, the Insular Telephone Network of Tenerife, which gradually evolved to an advanced integration model (at least for the time). The different stages of this process are described, and a comparison is made with the situation of the Telegraph Corps and the Telephone Company.

Keywords: Tenerife, Insular Telephone Network, telephonists, gender, integration. 
Siempre es un ejercicio arriesgado tratar de estudiar las mentalidades de una época a través del prisma de la sociedad actual. Esto resulta incluso más evidente cuando se pretende, por ejemplo, estudiar los procesos de incorporación de la mujer a una determinada actividad laboral, ya que hay que tener en cuenta los estereotipos compartidos por la sociedad. Estos se basaban en los roles de actividad predominante para las mujeres de cada época y poseían, por un lado, normas descriptivas y expectativas consensuadas respecto de las conductas esperables de cada género; por otro, normas prescriptivas que consagraban la inercia del mantenimiento de la división del trabajo por géneros. Así, a la mujer se le atribuían características de cuidado, calidez, amabilidad, sensibilidad y empatía que reflejaban el interés por el bienestar de otros, ya que socialmente su rol se encontraba principalmente relacionado con el cuidado y la protección de la familia. En cuanto al hombre, se le atribuían rasgos de asertividad, autonomía, actividad e independencia, reflejando conductas dominantes de mayor estatus y poder.

El peso de estos roles subyacentes tuvo su reflejo en el proceso de incorporación femenina a la actividad laboral en las compañías de telecomunicaciones, tanto en el caso de los servicios telegráficos como, posteriormente, en las primeras redes telefónicas ${ }^{1}$. Este nuevo mercado laboral tuvo un innegable impacto social, al posibilitar una cierta independencia económica y social para un colectivo de mujeres mayoritariamente jóvenes, con un cierto nivel formativo e inquietudes técnicas. Así se creó un nuevo nicho de empleo de alta capilaridad, ya que alcanzaba a muchos pequeños núcleos de población. Por el contrario, también se vio lastrado por la presencia de factores de discriminación salarial, laboral y social que supusieron, en muchos casos, el bloqueo de sus posibilidades de desarrollo personal. El efecto de estos condicionantes permitió que las mujeres pudieran incorporarse a actividades como la conmutación de líneas y la atención al cliente en las primeras redes telefónicas (trabajo que tendieron a desarrollar casi en exclusiva), así como a desempeñar papeles auxiliares en las redes telegráficas, basándose en una supuesta mayor paciencia, comprensión y cercanía. Esos mismos patrones limitaban su acceso a realizar turnos de noche, al reparto físico de los avisos y, por descontado, a puestos técnicos y de alta dirección, que se consideraban impropios de su condición. La incorporación de las mujeres se vio también teñida de un cierto paternalismo, ya que con la excusa de la protección social estas plazas, en ocasiones, se reservaban a viudas, hijas o hermanas de empleados, lo que no dejaba de ser una forma apenas edulcorada de machismo.

Además de estos factores sociológicos, no hay que menospreciar la importancia de las limitaciones motivadas por aspectos económicos. Las mujeres, en muchos casos, no formaban parte de las plantillas ordinarias de las empresas, por lo que no

* Instituto Universitario para el Desarrollo Tecnológico y la Innovación en Comunicaciones (IDeTIC), Universidad de Las Palmas de Gran Canaria.

** Departamento de Ciencias Históricas, Universidad de Las Palmas de Gran Canaria.

${ }^{1}$ Para los modelos de incorporación de la mujer a las empresas tecnológicas, véase LiparTITO (1994) у UEDA (2002). 
veían reconocidos derechos sindicales ni sociales. Además, había una tácita aceptación social de que su actividad podía recibir una retribución menor que la de un puesto equivalente desempeñado por un varón, al que se le suponía una responsabilidad familiar mayor. También se consideraba que su carrera laboral sería temporal, hasta llegar a un estatus más propio de su condición como eran el matrimonio y la maternidad, lo que limitaba sus posibilidades de promoción. Esta posible evolución se veía lastrada por la presencia de prejuicios sobre carencias de formación, incapacidad física o «falta de carácter» para desempeñar puestos directivos. La consecuencia global de esto era una "cosificación" del trabajo de la mujer en estas empresas (en algunos casos tenían incluso contratos dentro del capítulo de «materiales» en lugar de en concepto de "personal»), que se veía relegado a actividades extensivas en trabajo, pero poco intensivos en formación, lo que las convertía en víctimas principales del desarrollo tecnológico que automatizaba las redes y centrales, permitiendo a las empresas prescindir de esta fuerza de trabajo o minimizar su peso económico.

Finalmente, existía también una discriminación legal, que colocaba a las mujeres bajo la patria potestad primero de sus padres, y luego de sus maridos, limitando así sus derechos sociales. De este modo cabía preguntarse (y así se hizo incluso en sede parlamentaria, como se verá más tarde), para el caso de una central donde trabajaran juntos marido y mujer, si esta no debería obediencia a su cónyuge aun cuando ella ostentase un rango más alto, lo que menoscabaría el principio de jerarquía en el servicio. Atendiendo a estas consideraciones, se pueden establecer hasta tres situaciones dentro del proceso de integración laboral de las mujeres a las empresas de telecomunicación, aunque probablemente esto podría extenderse a otras situaciones en el proceso de incorporación femenina a la actividad en empresas y administraciones:

- Un primer modelo sería la incorporación a una escala propia y separada, en general con acceso limitado, lo que conllevaba la doble discriminación de unas retribuciones más reducidas y, al mismo tiempo, la ausencia de los derechos laborales y de posibilidades de promoción de las escalas y cuerpos generales. Ejemplos de esto serían las escalas de auxiliares telegráficos, o las telefonistas de las primeras compañías a finales del siglo xix y principios del xx.

- Una primera conquista sería la integración de las trabajadoras en las escalas regulares, si bien en este segundo estadio se mantendrían tablas retributivas y de actividad separadas de las de los varones. En ese caso la limitación de acceso a escalas y grados superiores se argumentaba con la falta de formación para desempeñar puestos técnicos -justificada, por ejemplo, por la falta de mujeres ingenieros-, o por la imposibilidad de desarrollar actividades de dirección vedadas al género femenino una supuesta falta de carácter. En ocasiones, pese a no existir una prohibición formal de acceso a ciertos puestos y escalas administrativas, las solicitudes presentadas por mujeres quedaban en un «limbo» administrativo donde no eran formalmente rechazadas, pero tampoco procesadas ni mucho menos aceptadas. Un ejemplo lo tenemos en la situación de las mujeres telegrafistas durante la II República y en las escalas administrativas de CTNE. 
- El siguiente modelo suponía la plena integración en escalas únicas, con igualdad de responsabilidades y retribuciones, para ciertos empleos dentro de las compañías, dándose ya el caso de mujeres que ocupan puestos en el escalafón por encima de sus compañeros varones, aunque se mantenían las limitaciones antes expuestas para el acceso a escalas superiores técnicas o administrativas. Este sería el caso de la Red Telefónica del Cabildo de Tenerife.

En los apartados siguientes se mostrarán las causas que posibilitaron que el mercado laboral de las, en ese momento, nuevas tecnologías se abriera a las mujeres. Luego se describe muy someramente la evolución histórica de la Red Telefónica Insular de Tenerife antes de ver la evolución del trabajo femenino en la misma, comparándola con el modelo de integración del servicio de Telégrafos.

\section{LOS PRIMEROS MODELOS DE INTEGRACIÓN}

La llegada de las tecnologías de telecomunicación (primero la telegrafía y poco después el teléfono) supuso una revolución no sólo desde el punto de vista de sus aplicaciones, sino también porque permitió crear una serie de nuevas oportunidades laborales que poco a poco se fueron abriendo a la incorporación de las mujeres. De la Peña $(2003)^{2}$ sitúa como causa la escasez de mano de obra masculina producida por la Guerra de Secesión en Estados Unidos, si bien en enero de 1870 el Journal Telegraphique ${ }^{3}$ sitúa ya esta presencia como algo habitual en muchos países europeos. En España este proceso se retrasó hasta las reformas de los cuerpos de telegrafistas de 1879, cuando un decreto regulador del servicio de telégrafos creó 400 estafetas telegráficas «limitadas» (con un único operador). En noviembre de 1880 se aprobó la contratación «en concepto de auxiliares del Cuerpo de Telégrafos a la mujer, hija o hermana del encargado de algunas [de estas] estaciones limitadas», cobrando "como jornal» cinco reales diarios, pero siempre "con cargo al capítulo de Material» ${ }^{4}$.

Para solicitar el puesto de trabajo la futura auxiliar tenía que demostrar «conocimientos de castellano, de lectura y escritura con buena ortografía [y] operaciones matemáticas sencillas», pero sobre todo puntuaba saber transmitir y reci-

2 De la Peña (2003), p. 97

3 Journal Telegraphique de 26 de enero de 1870, p. 1 y ss. se revisa la situación en los distintos países asociados a ITU. Se resalta que en los países nórdicos las mujeres eran contratadas como agentes en todas las oficinas telegráficas, en Italia y Francia lo hacían con restricciones y en Alemania y Bélgica sí eran familiares del empleado y trabajaban bajo su responsabilidad. Accesible en https:// historicjournals.itu.int/viewer/1296.

${ }^{4}$ Circular núm. 23 de la Dir. Gral. de Correos y Telégrafos de 23 de noviembre de 1880. En Revista de Telégrafos núm. 61, diciembre de 1880, p. 214. También puede consultarse Olıvé (2013), p. 78, y Crespo Gutiérrez (2015). Esta última, directora del Museo Postal y Telegráfico, ha estudiado en detalle la evolución laboral de este colectivo en España. 
bir en el sistema morse, amén de una «certificación de buena conducta y moralidad expedidos por el cura párroco y el alcalde de la localidad donde residan» ${ }^{5}$. La primera mujer fue contratada el 20 de enero de 1881, algo de lo que se señalaba «significa el reconocimiento de las necesidades del sexo femenino, y es una declaración [...] por la cual se reivindican las facultades femeninas y se atiende con especial cuidado a la educación de la mujer» ${ }^{6}$. Apenas cuatro meses después La Revista de Telégrafos $^{7}$ hablaba de "innovación en el servicio telegráfico" porque tenía contratadas ya once auxiliares femeninas, y admitía que los resultados estaban siendo buenos. Un año más tarde, en 1882, fueron admitidas cuarenta mujeres con un sueldo anual de 625 pesetas, significativamente menor que el de la categoría más baja de los telegrafistas varones (la de «aspirante»), que era de 1000 pesetas. Estas iniciativas oficiales del acceso de la mujer al trabajo fueron apoyadas también por la Asociación para la Enseñanza de la Mujer, que creó en 1883, en Madrid, la Escuela de Telégrafos para Mujeres, donde tras un ciclo de formación de dos años se obtenía el título de «telegrafista» ${ }^{8}$.

Inicialmente la reacción de sus compañeros varones fue de curiosidad (ya que su escaso número no amenazaba su posición), pero en las revistas profesionales pueden encontrase actitudes que van desde el machismo al paternalismo ${ }^{9}$, posturas que se trasladaron al Senado cuando en 1882 se debatió el proyecto de organización del Cuerpo de empleados de Comunicaciones ${ }^{10}$. El ministro de Gobernación, Venancio González y Fernández, justificaba que esta medida se tomó en principio desde un punto de vista meramente económico: «En España hemos comenzado un ensayo que está produciendo muy buen éxito. [...] sé permite a las esposas de los telegrafistas de ciertas Estaciones que puedan servir el aparato telegráfico, a fin de que el marido descanse algunas horas. Esto, como es consiguiente, ahorra el utilizar nuevos empleados, no obstante, la exigua gratificación de cinco reales diarios que se da a estas mujeres». Sin embargo, ahora se iba a dar un paso más allá, "pues se viene observando que el servicio prestado por ellas es más esmerado que el que realizan los maridos», si bien se introducía una clara discriminación al considerar que «el ingreso de esas mujeres no puede ser como el de los telegrafistas que lo verifican en el mismo Cuerpo, y como por otra parte también se requieren dotes de mando

${ }^{5}$ Este requisito era común a otras convocatorias; por ejemplo, para oficiales segundos se solicitaba, además de «la fe de bautismo legalizada en debida forma», «una certificación de buena conducta, expedida por la Autoridad». Gaceta de Madrid, n. ${ }^{\circ}$ 78, 18 de marzo de 1880, p. 707.

${ }^{6}$ Revista de Telégrafos, núm. 63, de 1 de febrero de 1881, p. 258. Se trataba de Josefa Álvarez Portela, cuya antigüedad era de 20 de enero de 1881, y esposa del oficial de la estación de Nava del Rey (Valladolid). La revista El Electricista de mayo de 1911 recoge una nota biográfica con motivo de su fallecimiento.

7 Revista de Telégrafos, n. ${ }^{\circ}$ 66, 1 de mayo de 1881, p. 307.

8 Ballardin (1989) y Crespo Gutiérrez (2015).

9 Para ilustrar este hecho, véase el debate del que se hizo eco la Revista de telégrafos, n. ${ }^{\circ} 76$, 1 de marzo de 1882 , p. 38.

${ }_{10}$ Diario de Sesiones del Senado, n. ${ }^{\circ} 83,24$ de abril de 1882, p. 1599 y ss. Debate recogido literalmente en Revista de Telégrafos, n. ${ }^{\circ} 78,1$ de mayo de 1882. p. 66. 
y aptitud legal para en muchos casos representar al Estado». También comentaba el ministro que «debían trabajar sólo en estaciones atendidas únicamente por mujeres» dado que consideraba que difícilmente podían ocupar puestos de responsabilidad entre varones. La réplica, favorable a integrar más a las mujeres en el servicio, la dieron los senadores Manuel Galdo y Conde de Casa-Valencia (Emilio Alcalá Galiano), mientras el senador Manuel Ortiz de Pinedo planteaba las incongruencias del sistema en el marco de la legislación vigente en España:

¿Cómo ha de resolverse ese problema sin relacionarlo en la ley del matrimonio y las leyes sobre la patria potestad, que ponen a la mujer bajo la autoridad del jefe de la familia? ¿Cómo se resuelve que una hija de familia mande a su padre, empleado de categoría inferior, o una mujer a su marido, de quien sea jefe administrativo?

Esto precisamente es lo que buscaba evitar el ministro «segregando» las oficinas atendidas por mujeres. Cuando el senador José Magaz planteó que, si bien la Ley no prohibía el acceso al cuerpo ordinario de telégrafos a la mujer, en la práctica no se concedía, por lo que las solicitantes quedaban en un limbo administrativo. Así quedó clara la motivación de la propuesta gubernamental:

¿Qué inconveniente, pregunta el Sr. Magaz, hay en que se ponga un artículo declarando el derecho de la mujer para ingresar en el Cuerpo de Comunicaciones? Pues yo creo que tiene más inconvenientes que ventajas, porque declarado ese derecho, ingresará la mujer en el Cuerpo de Comunicaciones por la misma puerta que los hombres, y por mucha violencia que haya luego para interpretar el artículo, hemos de tropezar con grandes dificultades para limitar sus derechos; mientras que por mi sistema, al admitirla a prestar un servicio, se pueden establecer mejor las condiciones en que ha de prestarlo (el subrayado es de los autores).

En 1884 la contratación se hizo extensiva a «mujeres solteras y viudas de telegrafista». Además, se reguló la categoría de auxiliar temporera de telégrafos a la que se podían incorporar las mujeres, si eran mayores de 16 ańos y pasaban el correspondiente examen, para realizar trabajos de oficina y emisión y recepción de telegramas ${ }^{11}$. Pese a que la experiencia había demostrado que el trabajo de la mujer en los servicios telegráficos era muy útil y representaba un ahorro al Estado, y aun reconociéndose que la mujer podría ejercer una jefatura dentro de Telégrafos y tener a su cargo a otras telegrafistas, este trabajo seguía viéndose como algo limitado: sólo podían prestar servicio de día completo y no permanente (por tanto, no en horario nocturno) y no tenían derecho a cobrar gratificaciones por transmisiones y recepciones de despachos. En cualquier caso, la posición del personal femenino en ese servicio no se formalizó hasta 1909, con la Ley de Bases para la reorgani-

${ }^{11}$ Circular núm. 37 de la Dirección General de Correos y Telégrafos, Sección Telégrafos, 3 de noviembre de 1883. En Revista de Telégrafos, n. ${ }^{\circ} 98,1$ de enero de 1884, p. 8. Esta se completa con la Circular núm. 13 de la misma Dirección y Sección de 23 de abril de 1884. 


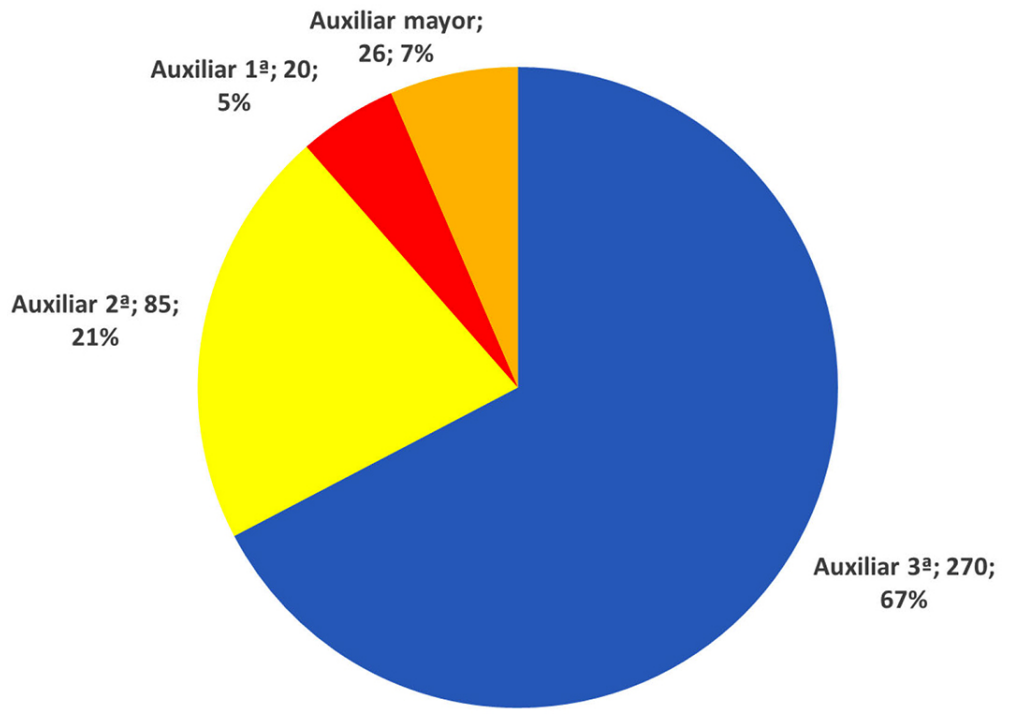

Gráfico 1: Distribución de las auxiliares femeninas por categorías (número y porcentaje). Fuente: Anuario telegráfico 1915, MPT.

zación de Telégrafos ${ }^{12}$; aunque esta regularización llegó con un retraso considerable respecto de los países de su entorno inmediato, finalmente otorgó a las mujeres telegrafistas la categoría de funcionarias. Las telegrafistas dejaron al fin de ser un «material» para poder ingresar en el personal de la administración. Aun así, se mantuvo la escala femenina separada, aunque con una estructura más compleja: en su cúspide figuraban las «auxiliares femeninas mayores» ${ }^{13}$, y después las auxiliares de primera, segunda y tercera. Las mujeres contratadas antes de esas primeras oposiciones quedaron como auxiliares mayores y las que habían aprobado se ordenaron atendiendo a los puntos obtenidos. El gráfico 1 muestra su distribución por categorías en 1915, mientras la tabla 1 ofrece una comparativa de sueldos entre las distintas categorías en 1911.

12 R.D. que aprueba el Reglamento orgánico del Cuerpo de Telégrafos, Gaceta de Madrid, n. ${ }^{\circ} 287,14$ de octubre de 1909, pp. 97-103. En él se indicaba (art. $\left.4 .^{\circ}\right)$ : «Constituyen el personal auxiliar los auxiliares de oficina, los auxiliares mecánicos, las auxiliares femeninas y el personal subalterno». Luego, el art. 117 definía la forma de ingreso de las auxiliares femeninas, que «se verificará mediante convocatoria» exponiendo luego las siguientes materias objeto del concurso.

${ }_{13}$ R.O. aprobando el Escalafón de auxiliares femeninas de Telégrafos. Gaceta de Madrid, n. ${ }^{\circ}$ 5, 5 de enero de 1911, pp. 75-76. La primera auxiliar mayor fue Rafaela González Pola García, «Rafaelita». 


\begin{tabular}{|c|c|c|c|}
\hline Puesto & SuEldo (PTAS.) & Puesto & Sueldo (PTAS.) \\
\hline Ordenanza $2 .^{\circ}$ & 725 & Oficial $2 .^{\circ}$ mecánico & 2000 \\
\hline Ordenanza $1 .^{\circ}$ & 850 & Auxiliar $4 .^{\mathrm{a}}$ contabilidad & 2000 \\
\hline Celador $2 .^{a}$ & 850 & Portero $2 .^{\mathrm{a}}$ & 2000 \\
\hline Celador $1 .^{a}$ & 850 & Auxiliar mayor & 2000 \\
\hline Capataz $3 .^{\text {a }}$ & 1000 & Oficial $1 .^{\circ}$ mecánico & 2500 \\
\hline Conserje & 1000 & Auxiliar $3 .^{a}$ contabilidad & 2500 \\
\hline Aspirante $2 .^{\circ}$ contabilidad & 1000 & Oficial $2 .^{\circ}$ & 3000 \\
\hline Auxiliar $3 .^{a}$ & 1000 & Auxiliar $2 .^{a}$ contabilidad & 3000 \\
\hline Carpintero & 1250 & Oficial $1 .^{\circ}$ & 3500 \\
\hline Capataz 2. ${ }^{a}$ & 1250 & Auxiliar $1 .^{a}$ contabilidad & 3000 \\
\hline Aspirante $1 .^{\circ}$ contabilidad & 1250 & Subdirector & 4000 \\
\hline Auxiliar 2..$^{a}$ & 1250 & Auxiliar mayor contabilidad & 4000 \\
\hline Capataz $1 .^{a}$ & 1500 & Director sección 2. ${ }^{\mathrm{a}}$ & 5000 \\
\hline Oficial $3 .^{\circ}$ mecánico & 1500 & Director sección $1 .^{\mathrm{a}}$ & 5000 \\
\hline Oficial $5 .^{\circ}$ & 1500 & Jefe de centro & 7500 \\
\hline Portero 2. ${ }^{a}$ & 1500 & Inspector & 8000 \\
\hline Auxiliar $5 .^{a}$ contabilidad & 1500 & Inspector general & 10000 \\
\hline Auxiliar $1 .^{a}$ & 1500 & Jefe de sección & 10000 \\
\hline Oficial $4 .^{\circ}$ & 2000 & & \\
\hline
\end{tabular}

El siguiente Reglamento Orgánico del Cuerpo de Telégrafos, en $1915^{14}$, normalizaba la presencia de la mujer en Telégrafos, aunque se seguían prefiriendo «las huérfanas, viudas, hijas y hermanas de los funcionarios de Telégrafos" y apartaba del servicio a las mujeres casadas que contrajesen «matrimonio con oficiales del Cuerpo de Telégrafos", ya que entonces "podrán ser destinados con sus maridos a estaciones que no sean limitadas ni permanentes, y únicamente en este caso podrán seguir en activo». En ese año, en Canarias, sobre un total de 75 funcionarios de telégrafos (sin contar personal de mantenimiento ni administrativo), había un $79 \%$ de varones y un $21 \%$ de mujeres (16), y entre estas había dos auxiliares de segunda y catorce de tercera. Por tanto, un $88 \%$ del personal femenino percibía retribuciones inferiores a la menor que recibía un telegrafista varón, diferencia incrementada desde 1912,

14 Real Decreto que aprueba el Reglamento orgánico del Cuerpo de Telégrafos, Gaceta de Madrid, n. ${ }^{\circ}$ 61, 2 de marzo de 1915, pp. 677-686. Las normas que desarrollaban este Reglamento recogían un uniforme de trabajo que consistía en una bata de color gris, cerrada por el cuello y los puños. En las vueltas del cuello llevaba el emblema de Telégrafos. 
ya que estos cobraban un complemento de residencia similar al de los funcionarios de Correos. Se trataba de una plantilla a todas luces insuficiente, sobre todo desde la creación de las centrales radiotelegráficas de Melenara y Cuatro Torres ${ }^{15}$, lo que obligaba a realizar turnos prolongados, incluso los fines de semana, haciendo su trabajo más penoso que el de sus equivalentes en la Península ${ }^{16}$.

Pero en ese momento Telégrafos ya había dejado de ser la única oportunidad laboral para las jóvenes. En los albores de la telefonía la compañía Bell había empleado en sus cuadros de conmutación a niños, siguiendo la tradición de las oficinas de telégrafos: los mayores (entre 15 y 17 años) se encargaban de la parte superior de los paneles de conexión, y los más pequeños (de 10 a 15 años), de los paneles inferiores hasta que la introducción de una legislación más severa contra el trabajo infantil redujo los beneficios de usar estos empleados ${ }^{17}$. Las crónicas de la época decían que, para establecer una llamada telefónica (algo definido entonces como una «algarabía»), se llegaba a necesitar cinco minutos ${ }^{18}$. El empleo de chicos adolescentes como operadores demostró ser uno de los fracasos más completos y consistentes del origen de las comunicaciones telefónicas. Arthur Clarke, en su descripción de esos primeros años de la conmutación, relaciona su efecto en términos de eficiencia con un proverbio chino: «Un chico es un chico, dos chicos equivalen a medio chico y tres chicos equivalen a nada ${ }^{19}$. Las referencias que se tienen de su rendimiento laboral recogen daños en los cuadros de conmutación, insultos a los suscriptores y que «era más habitual encontrarlos peleándose en el suelo que sentados en su banqueta realizando el trabajo preciso y repetitivo propio del operador de una centralita $»^{20}$.

Por eso, cuando se produjo la sustitución de los muchachos por mujeres jóvenes se consideró como una bendición. Las crónicas destacan «la voz tranquila, aguda, los dedos hábiles, la cortesía paciente y la atención, estas cualidades eran precisamente lo que el gentil teléfono requería en sus asistentes. Las chicas eran más fáciles de entrenar; más cuidadosas y eran mucho más propensas a dar la respuesta suave que aleja la ira $»^{21}$. Pero aparte de estas justificaciones, teńidas de un cierto paternalismo, la incorporación de mujeres a este trabajo tenía otras causas: estaba socialmente aceptado que cobraran menos que los varones por la misma actividad. Además, a las mujeres se les negaban los derechos sindicales y se esperaba de ellas que no tuvieran expectativas de progresar en su profesión. Por tanto, si bien es innegable que el desarrollo del servicio telefónico contribuyó de manera notable a la incorpo-

15 Pérez-Jiménez (2019).

16 Véase La Provincia, 4 de noviembre de 1911, p. 1.

${ }_{17}$ M. Schuman: «History of child labor in the United States - part 1: little children working» en Bureau of Labor Statistics. U.S. Dpmt. of Labor, accessible en https://www.bls.gov/opub/ $\mathrm{mlr} / 2017 /$ article/history-of-child-labor-in-the-united-states-part-1.htm.

${ }_{18}$ Recogido en HuUrdeman (2003), p. 191 y ss.

19 Clarke (1996), p. 192.

${ }^{20}$ Gleick (2013), p. 68

${ }^{21}$ Martín (1988). 
ración de la mujer a la vida laboral, también es cierto que se hizo de acuerdo a salarios y condiciones diferentes a los obtenidos por sus colegas varones.

Pero el halo de ser una profesión moderna, junto con la ausencia de un trato directo con el público, hicieron que ser telefonista se considerara un trabajo «honorable» para una mujer joven de clase media. Como decía un periódico de 1883: «Aquí hay una ocupación a la que ningún padre celoso podría poner reparos, el resultado es que una clase superior de jovencita puede obtenerse de la carrera de telefonista ${ }^{22}$. El entorno social aún consideraba que el matrimonio seguía siendo la carrera por excelencia de la mujer ${ }^{23}$, algo que en las clases medias podía llegar a ser traumático, ya que se esperaba que las hijas encontrasen maridos con recursos para que las mantuvieran «decentemente», lo que excluía trabajar fuera de casa o ejercer profesiones liberales y, por supuesto, necesitar ganar sueldo ${ }^{24}$. El trabajo se configuraba, así, como una etapa transitoria, no como una carrera profesional, para las muchachas de clase media que se concentraban en una serie de profesiones altamente feminizadas consideradas «respetables»: maestra, bibliotecaria o enfermera. Las telefonistas, y en menor medida las telegrafistas, se incluirían en este segmento.

No hay una fecha definitiva para la creación de la primera central de conmutación en España ${ }^{25}$, si bien en La Ilustración Española y Americana de enero de 1886 se publicó un grabado de la Estación Central de Teléfonos de Madrid, por lo que se puede aventurar que quizás datase de $1885^{26}$, aunque otros autores lo retrotraen a $1882^{27}$, desde el momento en que se aprobó la explotación e instalación de las primeras redes urbanas telefónicas en España. En ese grabado se contempla que sólo eran «señoritas telefonistas» la que realizaban estas tareas y, a partir de ese momento, todas las compañías que operaban en España pusieron en manos femeninas la responsabilidad de los cuadros de conmutación ${ }^{28}$. En un entorno donde se

22 Pall Mall Gazette, Londres, 6 de diciembre de 1883, p. 11, pero había una serie de requisitos. The Kansas City Star, 31 de diciembre de 1899, p. 3, recogía que "para ser una hello girl la solicitante debía ser menor de 30 ańos, medir al menos 1,68 de altura, tener un oído excelente, voz suave y temperamento angelical». Se preferían muchachas delgadas de brazos largos y de ascendencia irlandesa "por su capacidad de controlar su carácter admirablemente». Se denominaba Hello girl a las telefonistas en los países anglosajones, ya que las instrucciones las obligaban a saludar con un Hello a los clientes.

${ }^{23}$ Francos Rodríguez (1920), citado por García Basauri (1978).

24 SCANlon (1976), p. 54 señala que se consideraba perfectamente natural que una chica estudiara una carrera y no la practicara nunca. Por su parte, Margarita Nelken, en Nelken (1921), p. 64, se preguntaba, de acuerdo con esto, cuántos tomarían en serio a la mujer que pretendiera ejercer sus estudios de Derecho, dar clases en la Universidad o practicar como arquitecto. García Basauri (1978) señala que esta presión era menor en las clases populares, donde sí se asumía la necesidad de que la mujer aportase ingresos a la familia, que, desde luego, en las más acomodadas.

${ }^{25} \mathrm{Si}$ bien en La Habana la primera central de conmutación fue instalada en 1877.

26 Véase https://historiatelefonia.com/2015/07/31/telefonistas-en-espana/. Consultado el 18 de mayo de 2019. La central estaba en la calle Mayor núm. 1 de la capital.

27 UEDA (2002).

${ }^{28}$ La única excepción, obviamente, eran los telefonistas en guarniciones y unidades militares. 
decía que la mujer española sólo podía ser «reina, maestra o telefonista ${ }^{29}$, trabajar como "señorita telefonista» suponía un importante ascenso social. A pesar de ser un trabajo exigente, tenía más prestigio que otros trabajos desempeñados por mujeres en los talleres, las fábricas, el servicio doméstico o en las labores agrícolas, con mejores condiciones laborales y sueldo más elevado. El reconocimiento social a su trabajo y las relaciones con otras compañeras al margen del ámbito puramente familiar ofrecieron a estas trabajadoras un aura de modernidad, de modo que su figura pronto apareció en chistes, sainetes ${ }^{30}$, polkas y canciones, teatro y películas, creando una mitología social sobre "telefonista se casa con millonario al que conoce por teléfono».

Sin embargo, la realidad era muy distinta. Las operadoras eran la cara frente al público de las compañías y se les hacía responsables de muchos fallos producidos por sus deficiencias en equipos y tendidos ${ }^{31}$, por lo que tenían que escuchar cómo las increpaban los clientes y se enfrentaban a pérdidas de sueldo por sus quejas. Atendían paneles muy grandes (lo que producía errores y retrasos) y afrontaban turnos con horarios prolongados en salas mal ventiladas, con frío o calor (la calefacción sólo funcionaba para las telefonistas del turno de noche). El tamaño de los paneles hacía que tuvieran que trabajar muchas veces de pie y haciendo grandes contorsiones para poder unir las llamadas, trabajando en silencio, lejos de la imagen de "parlanchinas» que adquirieron, ya que en las centrales se sabía que estaba entrando una llamada porque se iluminaba una luz, no porque sonara ningún timbre. Las normas eran estrictas: vestían batas negras o azules ${ }^{32}$ y debían guardar silencio, tener actividad, ser lacónicas y amables, repetir los números y no escuchar conversaciones. Aun así, las quejas de las telefonistas provenían más de los bajos sueldos (unas dos pesetas diarias para la escala más baja ${ }^{33}$ ) y de la falta de horas de descanso que de las condiciones de trabajo ${ }^{34}$.

Los requisitos de acceso a la condición de telefonista cambiaron poco a lo largo del tiempo. En 1916 se especificaba que una telefonista tenía que ser «soltera, tener entre 15 y 25 años, contar con buena vista y oído y una moral intachable ${ }^{35}$;; como conocimientos, debían saber «las cuatro reglas básicas de aritmética elemental,

\footnotetext{
29 Se trata de una cita atribuida habitualmente a Concepción Arenal, pero al parecer es apócrifa.

${ }^{30}$ Paca la telefonista o el poder está en la vista, sainete en dos actos y tres cuadros, de Luis Fernández de Sevilla y Anselmo Carreño, con música de Enrique Daniel, de gran éxito en temporada de invierno de 1929 a 1930 en Madrid.

${ }^{31}$ El Duende, 7 de diciembre de 1913, p. 3. Pueden consultarse más testimonios de telefonistas en http://www.libropatas.com/libros-literatura/la-vida-secreta-de-las-senoritas-telefonistas/. Consultado el 1 de junio de 2019.

32 «Femeninas» en El Imparcial, 1 de diciembre de 1917, p. 3, reportaje de Magda Donato.

${ }_{33}$ En 1920 el sueldo base había subido un 50\%, La Voz, 12 de noviembre de 1920, p. 4.

${ }_{34}$ Ibidem.

35 Esto queda reflejado en el artículo 119 del R.D. «aprobatorio del adjunto reglamento para el establecimiento y explotación del servicio telefónico» de 9 de junio 1903, Gaceta de Madrid núm. 167, de 16 de junio de 1903, pp. 1016-1021, modificado en el R.D. de 30 de abril de 1907, Gaceta de Madrid, n. ${ }^{\circ} 125$, de 25 de mayo de 1907, p. 483.
} 
contar con una buena ortografía, unas cuantas cuestiones de geografía, un poco de historia y de cultura general y saber tomar dictado» (en realidad dos dictados, «uno deprisa y otro despacio ${ }^{36}$ ). Las pruebas de cultura general, aparentemente sencillas, suponían una verdadera selección, ya que el acceso de la población femenina a la educación básica era muy limitado, por lo que existían centros especializados para la formación de las candidatas ${ }^{37}$. También tenían que pasar una "prueba de envergadura" para asegurar que podían acceder a los extremos de su puesto (1,55 metros de distancia): un timbre sonaba si, extendiendo los brazos, conseguía tocar a la vez dos interruptores situados a una determinada distancia el uno del otro. Tras superar estos requisitos, luego completaban su formación con tres meses como alumnas en prácticas -no retribuidas- en una central, y entonces podían trabajar como operadoras en alguna de las compañías de teléfonos, al menos hasta casarse (en general, sólo las solteras o las viudas que hubiesen trabajado anteriormente en la empresa podían ser telefonistas). Las condiciones de trabajo eran muy duras: turnos de trabajo de al menos siete horas seguidas (y en centrales pequeñas hasta diez), con dos «emergencias» para ir al servicio y un descanso de un máximo de media hora para tomar un bocadillo o un café. La disciplina era casi militar ${ }^{38}$ y su vida laboral breve (unos tres años en media), ya que finalizaba al contraer matrimonio, lo que hacía inviable que las mujeres pudieran acceder a puestos técnicos o de dirección. Sin embargo, a lo largo de la década de 1920 este perfil fue, poco a poco, cambiando hacia la búsqueda de una mayor profesionalización, tanto por parte de las compañías, cuyo crecimiento demandaba mayor número de operadoras formadas, como por parte de las mujeres, cuyos horizontes laborales y personales se iban ensanchando.

\section{LA RED TELEFÓNICA INSULAR DE TENERIFE}

En ese entorno se produjo la creación de la Red Telefónica Insular de Tenerife. La situación de las redes telefónicas en Tenerife había llegado a principios de 1914 a tal nivel de deterioro que hacía necesaria una gran inversión en material y un proceso de homogeneización de sus redes. Estas estaban formadas por dos empresas principales: la Sociedad Telefónica de Tenerife en la zona de Santa Cruz y la Sociedad de Teléfonos de La Orotava en el valle homónimo, con centro en Puerto de la

36 Entrevista a Ricardo Charra, jefe de personal femenino de la Red Urbana de Teléfonos de Madrid al diario conservador La Acción. 4 de junio de 1916, p. 4. Esos requisitos, salvo la edad mínima, que ascendió a 16 años, son casi idénticos a los que menciona una de las pioneras, Celina Ribechini, en una entrevista recogida en El Diario de 26 de abril de 2017 (ed. digital).

${ }^{37}$ La Revista Ilustrada de Banca, Ferrocarriles, Industria y Seguros publicaba, a lo largo de 1910, un anuncio de Telefonía práctica, un manual que al precio de cinco pesetas «constituye un verdadero manual del telefonista, con cuyo estudio cualquier persona algo aplicada puede obtener los conocimientos necesarios para alcanzar una plaza o certificado de aptitud en cualquier centro telefónico».

${ }^{38}$ Todos estos datos proceden de entrevistas a telefonistas de la época, publicadas en https:// www.abc.es/cultura/20141023/abci-exposicion-telefonistas-201410221711.html. Consultado el $1 \mathrm{de}$ junio de 2019. 
Cruz, sin conexión entre ellas pero complementadas por una malla de líneas privadas (que se usaban también para servicio público) y líneas de carácter municipal entre las que había una amplia variedad de niveles de funcionamiento. La oportunidad de crear una red interurbana de carácter insular que interconectase los dos núcleos y diera servicio a las zonas no cubiertas vino dada por la confluencia de dos factores: la creación de los cabildos en 1912, que otorgaba el marco institucional para este despliegue, y la previsión (o imprecisión en su redacción) de la Ley La Cierva de $1907^{39}$ al permitir que «entidades» sin especificar pudieran hacerse cargo de estas redes, lo que posibilitó que nuevas realidades territoriales como la Mancomunitat de Cataluńa o los gobiernos insulares pudieran acceder a la titularidad de compañías prestatarias del servicio telefónico. El 2 de abril de 1914 el presidente del Cabildo, Eduardo Domínguez Alfonso, fue mandatado por la institución para formular el proyecto de una red telefónica insular ${ }^{40}$. El factor que nadie hubiera podido prever es que el 30 de julio de 1914 se produjesen los primeros disparos de la Gran Guerra, lo que dio al traste con estas previsiones cambiando radicalmente las prioridades de la institución insular. Por eso, aunque por real orden del Ministerio de Gobernación de 6 de diciembre de 1915 se autorizó la constitución de la Red Insular de Teléfonos de Tenerife, hubo un impasse de más de cinco años hasta que se pudo hacer efectiva la creación de la red.

Las primeras líneas de la compañía entraron en funcionamiento en septiembre de 1921. Y si bien originalmente la RTIT figuraba en su licencia original únicamente como una red interurbana, la propia evolución del servicio, las demandas de los municipios y las carencias de la red de las operadoras urbanas de Santa Cruz y el valle de La Orotava fueron creando nodos con una tipología diferenciada. En las ciudades cubiertas por las redes urbanas existentes (Santa Cruz, La Orotava, Puerto de la Cruz y La Laguna), se crearon centralitas para asegurar tanto el servicio interurbano como la conexión con los abonados de esas compañías. En las de tamaño intermedio (municipios como Icod, Tacoronte, Güímar o Garachico), la demanda social obligó a establecer redes urbanas, que a su vez sirvieron como nodos de conexión a otros puntos de servicio telefónico en zonas rurales ${ }^{41}$. Los mayores núcleos rurales fueron atendidos por subestaciones que operaban un número limitado de terminales (unos 5-6 como máximo), en general en los edificios de las propias centrales. Finalmente, pueblos menores o enclaves aislados tenían uno o dos terminales en locutorios, que desempeñarían un papel similar al de los teléfonos públicos de finales de siglo Xx.

${ }^{39}$ Ley que autoriza al gobierno para que proceda a plantear o desarrollar los servicios de radiotelegrafía, cables y teléfonos, de 26 de octubre de 1907. Gaceta de Madrid, 28 de octubre de 1907 , n. ${ }^{\circ} 301$, p. 359.

40 LACGCT 001-1913-1914, acta de la Comisión permanente de 2 de abril de 1914, p. 194. También puede encontrarse una reseña en La Opinión, 6 de abril de 1914, p. 2.

${ }_{41}$ Como se indica en la ya mencionada acta de 21 de julio de 1922, LACGCT 1921-1923, p. 288 y ss. 
A pesar de las dificultades iniciales, el modelo de la Red Insular resultó ser muy avanzado, sólo comparable en su nivel de penetración geográfica a los de la Mancomunitat catalana o al de la Diputación de Guipúzcoa ${ }^{42}$. En todo caso, resultó una red mucho más rentable social que económicamente; de hecho, no obtuvo beneficios de operación hasta 1926, y desde luego siempre estuvo muy lejos de poder recuperar el desembolso que supuso el tendido de la infraestructura inicial ${ }^{43}$. Esta búsqueda de la rentabilidad social, si bien era muy bien recibida en pueblos hasta entonces ais$\operatorname{lados}^{44}$, suponía unos costes de mantenimiento muy elevados, agravados porque el volumen de la inversión inicial y las dificultades para financiarla habían obligado a tomar acciones tan arriesgadas como bajar la calidad de los cables utilizados frente a los inicialmente previstos en la planificación de la red ${ }^{45}$. Esto, unido a la gran longitud de kilómetros de líneas tendidas para un volumen de abonados que nunca llegó a superar los 1000, probablemente hacía la red poco atractiva para la iniciativa privada, y fue una de las causas por las que la CTNE no se mostró demasiado interesada en su incautación cuando lo hizo con las demás redes a partir de 1924.

\section{LA EVOLUCIÓN DEL TRABAJO FEMENINO EN LA RED INSULAR}

La Red Telefónica Insular de Tenerife es un ejemplo paradigmático que sirve para ilustrar la evolución entre los distintos modelos de integración laboral de la mujer en los servicios de telecomunicación. En ella se puede observar el paso de la situación paternalista de considerar a la mujer casi como un sujeto de asistencia social a su integración en escalas propias para, posteriormente, pasar a trabajar, en algunos casos, en escalafones mixtos y con retribuciones indiferenciadas de las de sus compañeros varones. Aun así, desde la óptica actual, la situación de la mujer en la citada red durante la década de 1920 y hasta la Guerra Civil distaba mucho de la igualdad real, aunque era significativamente mejor que la de sus compañeras telegrafistas -y de operadoras de otras compañías telefónicas españolas- y similar a la de las operadoras en otros países más avanzados. Las causas de esta mejoría no

42 Calvo (2010).

${ }^{43}$ El periódico El Progreso, 18 de septiembre de 1926, p. 1 indicaba que «la explotación de este servicio hasta el año pasado ocasionaba pérdidas al Cabildo, pero ha aumentado tan considerablemente en estos últimos meses, que por primera vez se espera que este año produzca un superávit». Sin embargo, se refiere sólo a los gastos de explotación y no contempla la inversión en las redes (100 000 pesetas ese año, como consta en La Provincia de 4 de junio de 1925 p. 2) ni la amortización de las inversiones anteriores.

${ }_{44}$ Puede destacarse, por ejemplo, que tras la inauguración de la central de La Victoria de Acentejo, en octubre de 1922, incluso la muy conservadora Gaceta de Tenerife reseńa que «la Villa acordó dar el nombre de "Domingo Salazar" a la calle Real». Muy importante debía considerarse el mérito para desposeer al Rey de una calle y que el diario, de conocida trayectoria católica y conservadora, no lo criticara. Gaceta de Tenerife, 26 de octubre de 1922, p. 1.

45 LACGCT 1921-1923, p. 99, acta de 25 de agosto de 1921. 
deben atribuirse a que los responsables del Cabildo Insular de la época -todos varones- fuesen un grupo de ardorosos sufragistas, dispuestos a arrojarse a los pies de los caballos en el hipódromo de Epsom ${ }^{46}$, sino a una serie de circunstancias socioeconómicas en las que entraremos a continuación.

Cuando, tras la Gran Guerra, se pudo por fin retomar con más visos de éxito el proyecto de tendido de la Red Insular, se hizo necesario prever la dotación de personal para realizar la conmutación de llamadas, de la misma forma que ya se realizaba en las dos redes existentes: las sociedades de teléfonos de La Orotava y de Tenerife (Santa Cruz). Las primeras referencias a la necesidad de cubrir estas plazas mencionan que «[dado que] la Corporación debe evitar, en la medida que le sea posible, la creación de clases pasivas», estimaba que las primeras plazas para proveer de telefonistas a la red se entregaran a personas en estado de necesidad, como era el caso de «las señoritas María, Matilde y Carmen Gutiérrez, huérfanas del Oficial Mayor, que fue de este Cabildo D. Pablo Gutiérrez y González», que habían presentado una instancia al Cabildo «en solicitud de que se les conceda una modesta pensión para atender a su subsistencia ${ }^{47}$.

Sin embargo, la necesidad de contar con personal formado en el manejo de los cuadros telefónicos, con buena dicción y capacidad de llevar las cuentas de las estaciones, pronto obligó a convocar oposiciones para este cuerpo ${ }^{48}$, especificando que las candidatas, además de estudiar el temario especificado, debían ser «españolas, mayores de 15 años de edad y menores de 35", presentar certificación de no padecer enfermedad infectocontagiosa y, si eran menores de edad, autorización del padre o tutor legal. En esa primera convocatoria el modelo de acceso era mixto, ya que de las veinte plazas disponibles «se cubrirán únicamente diez y nueve, por estar reservada la restante para la huérfana de un empleado municipal» cuya categoría se determinaría por examen. Las retribuciones, para las tres categorías de telefonistas (que se escalafonaban según los resultados de la oposición) eran 1500, 1200 y 900 pesetas/año para las telefonistas de primera, segunda y tercera respectivamente ${ }^{49}$, además de proveerlas de uniformes.

En las convocatorias subsiguientes la oposición se consagró ya como la única vía de acceso a los puestos de telefonistas que se asignaban a las distintas centrales (inicialmente cuatro «oficiales telefonistas» en Santa Cruz, tres en La Orotava, Icod o Garachico y dos en Los Silos ${ }^{50}$ ), siempre a cargo de una oficial primera. Por

${ }^{46}$ Allí falleció Emily Davison por intentar mostrar una pancarta delante de un caballo de la cuadra del rey Jorge $\mathrm{V}$ el 4 de junio de 1913.

47 LACGCT 1919-1921, acta de 4 de septiembre de 1919, p. 106.

${ }_{48}$ LACGCT 1919-1921, acta de 2 de junio de 1920, p. 227.

49 El Progreso, 21 de junio de 1920, p. 3. Estos sueldos eran más bajos que los equivalentes en Madrid. En El Liberal, 21 de enero 1911, p. 4, se recogía ya que las telefonistas ganaban entonces 1200 pesetas de sueldo anual.

50 El reparto de esas 19 plazas (la vigésima quedó desierta) era 5 de oficial 1. a 4 de oficial $2 .^{a}$ y 10 de oficial 3. ${ }^{a}$, ocupándose los puestos en función de la calificación obtenida, en LACGCT 1919-1921, acta de 15 de febrero de 1921, p. 365. Allí también se indicaba que el método de acceso sería sólo por oposición. 
el contrario, cuando se convocaron las plazas de celadores, mecánicos y capataces, que estaban a cargo del tendido y mantenimiento de los circuitos y centrales, se reservaron a varones (justificándolo por su perfil técnico y la necesidad de «subir a los postes»), al igual que las plazas de repartidor, encargados de llevar los avisos de llamada o los telefonemas, reproduciéndose así el esquema vigente en Telégrafos ${ }^{51}$. También quedaban inicialmente vedados a las mujeres los puestos de administración y gestión y, por supuesto, los de ingeniero y dirección.

Para poner en situación estos datos, hay que tener en cuenta que el requisito de acceso por oposición, en una población femenina en Canarias con tasas de analfabetismo de alrededor del $50 \%{ }^{52}$, era por sí muy limitante. La tasa de soltería estaba por debajo del $20 \%$ para los grupos de edad a partir de los 25 años $^{53}$, lo que unido a lo anterior podría explicar por qué, a diferencia de la mayoría de las compañías telefónicas de la época, no se cerraba el acceso a mujeres casadas a la RTIT, ya que eso hubiera reducido mucho la base social de posibles candidatas a telefonista. Para ver la repercusión de la creación de esta nueva oferta de empleo puede consultarse su incidencia sobre la tasa de actividad femenina en Canarias. Esta, comparada con la masculina y siempre atendiendo sólo a trabajos retribuidos, estaba en una relación alrededor de 80\%-20\%, y normalmente centrados en trabajos de baja cualificación (véase gráfico 2). Esto se daba a pesar de que la emigración había "feminizado» la población en Canarias, con un porcentaje de desproporción entre sexos que llegó en algunos casos al $15 \%{ }^{54}$. Sin embargo, y para dar idea de su creciente importancia, pese a que en los datos de 1930 se registra un descenso en el número de trabajadoras remuneradas, se da también un significativo aumento, tanto en valor absoluto como porcentual, en el conjunto del empleo del grupo de «administración» en el que se incluirían los empleos en la RTIT y Telégrafos, y de «otros» donde estarían los de CTNE. Dado que la RTIT contaba con entre 130 y 150 telefonistas, se le podría atribuir la responsabilidad directa de casi un 50\% del crecimiento en ese segmento.

Junto con este personal, que puede definirse como dentro de las escalas ordinarias, y dado que el modelo de negocio de la RTIT pretendía alcanzar todo el territorio insular, apareció la necesidad de atender a oficinas en locales aislados «en lugares cuya importancia no permite la presencia de una telefonista». Para ello se recurre a personas «caracterizadas» como maestras, dueños de bares, encargados de los fielatos etc., instalándose el teléfono en su casa o local y haciéndose cargo del servicio (atender las llamadas, repartir avisos, llevar la contabilidad). Estos, que podían ser varones o mujeres en igualdad de condiciones (de hecho, aproximadamente un tercio de los encargados de estas estaciones -o más propiamente, locutorios o teléfonos

51 Para las plazas de celadores, véase LACGCT 1919-1921, acta de 9 de junio de 1921, p. 38, mientras que para las de repartidores, puede consultarse la ya citada acta de 2 de junio de 1920 .

52 Martín Ruiz (1985), pp. 485 y 793.

53 Ibidem, p. 790.

54 Ibidem, p. 436. 


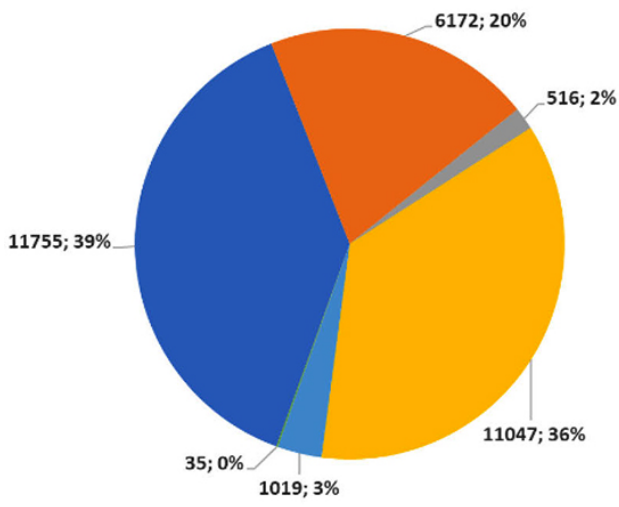

Agricultura y pesca
Industria y Manufactura
Comercio

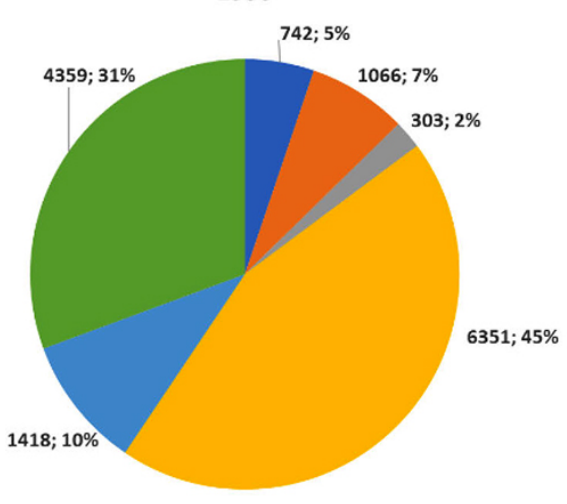

Servicio Doméstico Administración y servicios culturales Otros

Gráfico 2: Distribución por sectores de la población activa femenina en trabajos retribuidos en Canarias (número y porcentaje). Fuente: Álvarez (1980).

públicos- eran mujeres), percibían una retribución que podía alcanzar el 25\% de la recaudación, con un mínimo de 50 pesetas y un máximo de 75 pesetas mensuales ${ }^{55}$.

El siguiente paso de la evolución laboral fue la creación de un nuevo puesto denominado «jefa de estación», al que podían acceder las telefonistas al igual que sus compañeros masculinos. Esto indica dos aspectos que es necesario destacar: las telefonistas estaban abriéndose a iniciar una trayectoria laboral en la empresa, a pesar de que a priori podía presumirse que el trabajo de telefonista era algo transitorio hasta el matrimonio. Como ya se ha indicado, la soltería de las operadoras no fue nunca un requisito de trabajo en la RTIT, al contrario que en la mayoría de las compañías, o posteriormente en Telefónica. El segundo aspecto era la asunción de mayores responsabilidades que ese puesto conllevaba, y que no sólo cubría la atención al público, sino la contabilidad de la estación, gestiones con los proveedores, etc., lo que denota asimismo una salida de los roles asignados hasta ese momento a las mujeres en la RTIT ${ }^{56}$. A cambio, las condiciones de trabajo eran draconianas: las oficiales hacían diez horas diarias salvo los sábados, que tenían una jornada «redu-

55 LACGCT 1921-1923, acta de 12 de septiembre de 1921, p. 104. Para las retribuciones LACGCT 1921-1923, acta de 16 de febrero de 1922, p. 180.

56 LACGCT 1921-1923, acta de 21 de julio de 1922, p. 288. Estas primeras jefas de estación fueron, en Santa Cruz, Amparo Díaz Samson; en La Orotava, Clotilde Rodríguez González; en Güímar, África Celestina Suárez; en Icod, Pastora Martín Méndez, y en Garachico, María Dolores Dorta Santana. 
cida» de ocho, para un total de cincuenta y ocho horas semanales, mientras que los jefes de estación hacían turnos de hasta catorce horas diarias.

\begin{tabular}{|c|c|c|}
\hline \multicolumn{3}{|c|}{ TABLA 2: RETRIBUCIONES EN RED TELEFÓNICA INSULAR DE TENERIFE EN 1922* } \\
\hline Escala & Grado & RETRIBUCión ANUAL (PESETAS) \\
\hline \multicolumn{2}{|l|}{ Escala superior ${ }^{* *}$} & 5000,00 \\
\hline \multicolumn{2}{|l|}{ Jefe de estación } & 2100,00 \\
\hline Personal de administración & $\begin{array}{l}\text { Oficiales } \\
\text { Escribientes }\end{array}$ & $\begin{array}{l}2000,00 \\
1760,00\end{array}$ \\
\hline \multirow{3}{*}{ Telefonistas } & Primera & 1500,00 \\
\hline & Segunda & 1200,00 \\
\hline & Tercera & 970,00 \\
\hline \multirow{2}{*}{ Mantenimiento } & Capataces & 2000,00 \\
\hline & Celadores & 1760,00 \\
\hline \multicolumn{2}{|l|}{ Repartidores } & 547,50 \\
\hline
\end{tabular}

Para poner estos datos en contexto, un hombre con categoría de maestro cobraba en Santa Cruz de Tenerife una media de 0,86 pesetas/hora -con jornadas semanales de entre 48 y 52 horas- y una mujer entre 0,52 y 0,39 pesetas $/$ hora $^{57}$. Trasladando los datos de la tabla 2 , una jefa de estación estaría en 0,91 pesetas/hora, y las oficiales de primera, segunda y tercera estarían en 0,$65 ; 0,52$ y 0,42 respectivamente. Esto muestra unos sueldos competitivos dada la situación de la época, y significaría un factor de atracción importante a la hora de incorporar personal formado.

En una central típica de esos primeros años de la década de 1920, con un turno de tres personas, una atendía las conversaciones, otra anotaba las conferencias, mientras otra, que solía ser el jefe de estación, llevaba la taquilla y la administración de la oficina. En todos los casos había una clara insuficiencia de personal, ya que para una central de tamańo medio se hubiesen requerido unas diez personas, repartidas en varios turnos, cuando lo habitual es que hubiese un turno de tres operadores y otro de dos ${ }^{58}$. A estos problemas se añadía que la propia Corporación requería la movilidad geográfica de sus empleadas, a medida que se iban creando nuevas estaciones y se pretendía dotarlas de personal experimentado. En las condiciones de transporte de la época, un traslado casi siempre suponía la necesidad de buscar un nuevo domicilio y la imposibilidad de atender las cargas familiares que, en general, seguían soportando las mujeres, lo que motivaba continuas peticiones de baja temporal o definitiva en el servicio por parte de muchas de las afectadas. Esto

57 Millares Cantero (2011) tomo iI, p. 250.

58 LACGCT 1921-1923, acta de 24 de mayo de 1922, p. 238. 
se trató de paliar a partir de 1921, de forma algo timorata, mediante la dotación a sus operadoras de 100 pesetas a principio de cada año «de una sola vez, en caso de traslado, para pagar traslado de domicilio y ajuares", algo que el Cabildo veía como «un deber de humanidad y [...] el propio interés de la Corporación así lo exige „59. De nuevo, esto desmiente la pretendida temporalidad, y casi "amateurismo" que los estereotipos suponían a las empleadas femeninas en este tipo de empleos, quedando patente que, bien al contrario, un número creciente de ellas buscaba convertir este trabajo en una profesión.

Por lo que respecta a los sueldos, el escalafón más bajo de telefonistas, de tercera, cobraba en el periodo 1922-1928 algo menos que las telegrafistas de su misma categoría, mientras que las de segunda y de primera tenían retribuciones equivalentes a las de las auxiliares correspondientes de telégrafos. Las jefas de estación llegaban a las 2100 pesetas anuales, retribución superior a la de un oficial mayor de ese cuerpo. Este sueldo, en particular, superaba a los de la escala administrativa, en ese momento ocupada exclusivamente por varones, aunque quedaba lejos de los de las escalas superiores (ingenieros, directivos) a los que tampoco tenían acceso las mujeres, con la excusa (fundada en el caso de los ingenieros) de la falta de formación, ya que no había mujeres que hubiesen ingresado en las escuelas de ingeniería en esa época. A partir de septiembre de 1922, la normativa interna del Cabildo consagraba en las escalas de telefonistas, celadores, mecánicos y administrativos «el modelo de acceso por oposiciones, perdiendo su antigüedad si no se presentan a los concursos» ${ }^{60}$. A esta prueba se unía un periodo obligatorio (y no retribuido) de prácticas «en la estación de la localidad de residencia» que debían ser aprobadas por la jefa de estación ${ }^{61}$. Esta mejor formación y progresiva profesionalización hizo que, desde finales de la década de 1920, se fuera normalizando la presencia femenina, además de como operadoras, como administrativas, cobrando en general el mismo sueldo y con las mismas condiciones de trabajo que sus compañeros varones ${ }^{62}$. Para el año 1930 esa presencia estaba plenamente normalizada, y no sólo en los escalones más bajos de gestión, lo que hizo necesario un cambio normativo que se aprobó en octubre de $1931^{63}$, poco después de instaurada la II República.

59 LACGCT 1921-1923, acta de 12 de septiembre de 1921, p. 100. Estos cambios eran constantes y, en muchos casos, contra la voluntad de las telefonistas. Un ejemplo de estos traslados se refleja en Gaceta de Tenerife, 28 de noviembre de 1926, p. 2.

${ }^{60}$ LACGCT 1921-1923, acta de 5 de septiembre de 1922, p. 328.

${ }^{61}$ LACGCT 1924-1926, acta de 16 de agosto de 1924, p. 41.

${ }^{62}$ LACGCT 1928-1929, acta de 31 de mayo de 1928, pp. 119-120.

${ }^{63}$ LACGCT 1931-1932, acta de 14 de octubre de 1931, pp. 16-18. 


\section{LOS CAMBIOS DE LA II REPÚBLICA}

La proclamación de la República trajo algunos cambios en la legislación que, sin embargo, no llegaron a ser determinantes. Por ejemplo, se mantuvo la situación heredada del Estatuto de funcionarios de $1918^{64}$ que reconocía el derecho de la mujer a trabajar en la administración pública como auxiliar, pero manteniendo la discriminación salarial respecto del hombre. El nuevo régimen trajo el cambio de la denominación del personal femenino de «auxiliares» a «telegrafistas», integrándose en una escala común, aunque manteniendo la diferencia de sueldo con los varones ${ }^{65}$, diferencia que afectaba incluso al reconocimiento de las horas extraordinarias: mientras los hombres cobraban dos pesetas con cincuenta céntimos la hora, las telegrafistas percibían una peseta. Sin duda estas condiciones laborales no eran un buen reclamo para la incorporación laboral de mujeres en un momento en que tímidamente se abrían otras puertas para su trabajo; de ahí que en 1936, de un total de 849 plazas, 90 estuvieran vacantes sin que hubiera ingresado ninguna mujer desde 1918. La regulación republicana introdujo mejoras, no sólo en los sueldos, sino en las condiciones laborales de las mujeres en Telefónica: por ejemplo, las telefonistas ya no perdían su trabajo al casarse o al tener hijos; al contrario, las operadoras de CTNE tenían ahora incluso subsidio de maternidad ${ }^{66}$. Sin embargo, las mujeres seguían concentradas en un pequeño número de puestos en la compañía y había una nueva amenaza en el horizonte: la automatización que amenazaba la continuidad de sus empleos ${ }^{67}$ (véase tabla 3). Eso no compensaba la aparición de otras posibilidades laborales como ser telefonista en hoteles, hospitales o en grandes empresas.

\begin{tabular}{|c|c|c|c|}
\hline \multicolumn{4}{|c|}{ TABLA 3: CATEGORÍAS DE PERSONAL DE CTNE HACIA 1935* } \\
\hline \multicolumn{4}{|c|}{ Categorías } \\
\hline \multirow{3}{*}{ Jefes } & $1 .^{a}$ & \multirow{2}{*}{ Oficial administrativo } & 1. ${ }^{\mathrm{a}}$ \\
\hline & $2 .^{a}$ & & $2 .^{a}$ \\
\hline & $3 .^{a}$ & \multirow{2}{*}{ Auxiliar administrativo } & $1 .^{a}$ \\
\hline Jefes de negociado & & & 2. ${ }^{\mathrm{a}}$ \\
\hline Ayudante de explotación & & \multirow{2}{*}{ Jefa telefonista } & $1 .^{\mathrm{a}}$ \\
\hline \multirow{2}{*}{ Operador técnico } & $1 .^{a}$ & & $2 .^{a}$ \\
\hline & $2 .^{a}$ & \multirow{2}{*}{ Vigilante telefonista } & $1 .^{\mathrm{a}}$ \\
\hline \multirow{2}{*}{ Mecánicos } & $1 .^{a}$ & & $2 .^{a}$ \\
\hline & $2 .^{a}$ & Telefonista & $1 .^{\mathrm{a}}$ \\
\hline
\end{tabular}

${ }^{64}$ Ley de bases acerca de la condición de los funcionarios de la Administración civil del Estado de 20 de julio de 1918. Gaceta de Madrid núm. 205, 24 de julio de 1918, pp. 222-225.

${ }_{65}$ De hecho, sus sueldos llevaban congelados desde 1922.

${ }_{66}$ Nuevo Mundo de 15 diciembre del 1933, p. 23.

${ }^{67}$ Lo que situó a las mujeres en primera línea de la gran huelga de 1931 de Telefónica. 


\begin{tabular}{|c|c|c|c|}
\hline \multirow{2}{*}{ Capataz } & $1 .^{\mathrm{a}}$ & Telefonista & $2 .^{a}$ \\
\hline & $2 .^{a}$ & Conserjes & \\
\hline Celador-empalmador & & Ordenanzas (masc. y fem.) & \\
\hline \multirow{2}{*}{ Empalmador } & $1 .^{\mathrm{a}}$ & Guardas & \\
\hline & $2 .^{a}$ & Personal femenino de limpieza & \\
\hline \multirow{3}{*}{ Celador } & $1 .{ }^{\mathrm{a}}$ & Conductores & \\
\hline & 2. ${ }^{a}$ & Ayudantes & \\
\hline & & Peones & \\
\hline
\end{tabular}

* Marcadas aparecen las categorías accesibles al personal femenino. Fuente: elaboración propia a partir de anuarios de telefónica y CALvo (2010).

La reforma de la normativa de la Red Telefónica Insular de Tenerife formalizó el acceso a la escala administrativa e introdujo un cambio en los ascensos dentro de esta escala y la de telefonistas (denominadas ahora "operadoras»). Hasta ese momento en la escala administrativa los ascensos se producían sólo por antigüedad, pasando de una a otra categoría cada cinco años, lo que hubiera producido, como indicaba el propio Cabildo "que con el tiempo todos [los administrativos fuesen] oficiales de primera, perjudicando al servicio». Tras la reorganización de la escala administrativa esos ascensos se convertían en complementos salariales de 500 pesetas por cada quinquenio de antigüedad, dejando los cambios de categoría a criterios de mérito en función de las vacantes producidas. Si los cambios se producían sólo por antigüedad, los administrativos varones, con más ańos en la empresa, hubieran copado en exclusiva los puestos más altos, pero así se abrió una pequeña ventana para el ascenso de mujeres dentro de esta escala. Sirva como ejemplo el caso de un concurso para «llevar la administración del conteo de tráfico cursado, entre las operadoras», puesto para el que resultó seleccionada la auxiliar segunda de administración Adela González y González ${ }^{68}$.

La importancia de este cambio es que la escala de operadoras iba perdiendo progresivamente peso dado el proceso de automatización de las centrales, de forma que la reforma abría a estas empleadas la posibilidad de seguir trabajando dentro de la empresa. Las consecuencias de no llevar a cabo acciones similares condujeron a su progresiva «desfeminización» en el periodo comprendido entre 1945 y 1970, como recoge Calvo ${ }^{69}$. En efecto, durante esos ańos en Telefónica «aumentó la tasa de masculinidad en el conjunto de la empresa, situación compatible con una alta concentración de mujeres en los departamentos comercial y de tráfico». La mayoría de las mujeres empleadas en Telefónica en 1970 tenía menos de 25 años, y por tanto no habían desarrollado ningún tipo de carrera profesional en la compañía. Muchas de las de mayor edad seguían destinadas a ser operadoras en las pequeñas centralitas rurales que aún pervivían, y siempre pendientes de una posible automa-

${ }^{68}$ LACGCT 1932-1934, acta de 17 de abril de 1933, p. 171.

${ }^{69}$ Calvo (2010), p. 389. 
tización que significara su cierre, o a atender llamadas comerciales o posibles quejas o reclamaciones por parte de los usuarios.

Volviendo a la reforma de 1931 en la RTIT, y en lo que respecta a las operadoras, se suprimía la categoría inferior, que se asimilaba a la escala inmediatamente superior, se subían los haberes de las restantes categorías y se añadía un complemento salarial de un $10 \%$ cada cinco ańos de antigüedad (véase tabla 4).

\begin{tabular}{|c|c|c|c|}
\hline \multicolumn{4}{|c|}{$\begin{array}{l}\text { TABLA 4: ESCALAS RETRIBUTIVAS DE LA RTIT EN } 1931 \text { (OPERADORAS, } \\
\text { ADMINISTRACIÓN, MANTENIMIENTO Y REPARTO) }\end{array}$} \\
\hline & EMPLEOS & $\begin{array}{l}\text { SUELDO ANUAL } \\
\text { (PTAS.) }\end{array}$ & $\begin{array}{l}\text { AUMENTO POR } \\
\text { QUINQUENIO }\end{array}$ \\
\hline Jefa de estación & & 2600 & $10 \%$ \\
\hline \multirow{4}{*}{$\begin{array}{l}\text { Personal de } \\
\text { administración }\end{array}$} & Oficial $1 .^{\circ}$ & 2500 & 500 \\
\hline & Oficial 2.a & 2200 & 500 \\
\hline & Auxiliar $1 .^{\mathrm{a}}$ & 2000 & 500 \\
\hline & Auxiliar 2.a & 1900 & 500 \\
\hline \multirow{2}{*}{ Operadoras } & Primera & 1900 & $10 \%$ \\
\hline & Segunda & 1500 & $10 \%$ \\
\hline \multirow{3}{*}{ Mantenimiento } & Capataces mecánicos & 3000 & $10 \%$ \\
\hline & Capataces mecánicos de central automática & 5100 & $10 \%$ \\
\hline & Celadores & 2500 & $10 \%$ \\
\hline Repartidores & & 685 & \\
\hline
\end{tabular}

Fuente: LACGCT.

Sin embargo, pese a estos rasgos de modernidad, el panorama en la RTIT distaba mucho de ser ideal. En primer lugar, se mantenía una figura tan anacrónica como la de las «suplentes» en las estaciones menores, que cobraban su retribución directamente de las operadoras de plantilla en caso de enfermedad o imposibilidad de desempeñar su función ${ }^{70}$. También seguía presente la discriminación global que mantenía fuera del acceso femenino las escalas mejor retribuidas: no sólo la de dirección e ingeniería, sino las de mantenimiento, especialmente en aquellos puestos especializados como los destinados a sostener las nuevas centrales automáticas. Finalmente, había una forma de discriminación más sutil, como mantener permisos diferenciados para hombres y mujeres en caso de matrimonio, en función de su supuesta «mayor responsabilidad en la organización del hogar»»1.

${ }^{70}$ LACGCT 1932-1934, acta de 10 de noviembre de 1933, pp. 362-363. Sin duda esta posición dejaba muy en entredicho la «responsabilidad social» de la empresa en esos ańos.

71 En LACGCT 1932-1934, acta de 12 de junio de 1933, p. 227 se recogen permisos de 45 días para matrimonio en el caso de trabajadoras femeninas. 


\section{CONCLUSIONES}

¿Qué causas pueden apuntarse para que la Red Telefónica del Cabildo de Tenerife fuera un modelo socialmente más avanzado que otras empresas telefónicas de la época? La RTIT era una empresa pequeña (algo más de cien empleados, sin contar repartidores y personal en los locutorios rurales), de titularidad pública y de alta capilaridad en su desarrollo territorial, que se movía dentro de una política orientada más al mantenimiento del servicio -rentabilidad social- que al beneficio económico. El crecimiento acelerado del periodo 1921-24 supuso una enorme tensión en la necesidad de contratar -y posteriormente mantener- el personal capacitado. Además, la empresa debía cubrir toda una serie de estaciones en pequeñas poblaciones, lo que se sumaba a las dificultades para desplazar al personal, obligándola a ofrecer unas condiciones de trabajo dignas para sus empleados. Es de destacar también que la flexibilidad en el acceso a otras escalas profesionales permitía disminuir la tensión dentro de la compañía, sobre todo entre aquellas personas que se podían ver afectadas por la modernización de la red.

Todo ello configuraba un modelo diferenciado de lo que era habitual en el sector durante ese periodo, y que se mantuvo hasta la desaparición de la Red Insular en 1938, con su forzosa incorporación al monopolio estatal ${ }^{72}$. Esto configuraba a la RTIT como un caso particular, y más avanzado desde el punto de vista social y de integración femenina en la operativa general de la compañía. Evidentemente, este desarrollo fue truncado, al igual que en tantos otros aspectos, como resultado de la Guerra Civil, que supuso un lapso de cuarenta ańos en el proceso de obtención de nuevos derechos y posibilidades laborales para la mujer, así como en su integración en las compañías de telecomunicación.

Recibido: 16-6-2019; ACEPTADO: 11-6-2020

72 Ese mismo año, en la zona sublevada se promulgó el «Fuero del Trabajo», en el que se hablaba de «liberar a la mujer casada del taller y de la fábrica», con lo que se introdujo la condición de que las mujeres en el servicio de telefonía debían ser solteras o viudas. $B O E$ de 10 de marzo de 1938, n. 505 , p. 6178 y ss. 


\section{REFERENCIAS}

Álvarez, M. (1980). Estructura social de Canarias II. La reproducción del subdesarrollo, Las Palmas. CIES.

Ballardin, P. (1989). «La educación de la mujer española en el siglo XIX», Historia de la educación: Revista interuniversitaria, $n .^{\circ} 8: 245-260$.

Calvo, A. (2010). Historia de telefonica-1924-1975, Fundación Telefónica.

Clarke, A. C. (1996). El mundo es uno, Ediciones B.

Crespo Gutiérrez, M.V. (2015). «Las mujeres telegrafistas: pioneras en la administración». Reseña de la conferencia en el aula cultural del Ministerio de Fomento, 17 de mayo de 2015.

De la Peña, J. (2003). Historias de las Telecomunicaciones. Cuando todo empezó, Madrid, Editorial Ariel.

Francos Rodríguez, J. (1920). La mujer y la política españolas, Madrid, Librería de los Sucesores de Hernando.

GARCía BASAURI, M. (1978). «Una aproximación al primer movimiento feminista español: La mujer en el reinado de Alfonso XIII", Tiempo de Historia, núm. 46, pp. 36-42.

Gleick, J. (2013). «La información: Historia y realidad» (Serie Mayor). Ed. Crítica.

Huurdeman, A. (2003). "The Worldwide History of Telecommunications», John Wiley \& Sons.

Lipartito, K. (1994). «When Women Were Switches: Technology, Work, and Gender in the Telephone Industry, 1890-1920», The American Historical Review, vol. 99, n. ${ }^{\circ} 4$. (octubre), pp. 1075-1111.

Martín Ruiz, J. F. (1985). Dinámica y estructura de la población de las Canarias Orientales (siglos XIX y XX), 1982, tesis doctoral, Universidad de La Laguna.

Millares Cantero, S. (2011). Historia Contemporánea de Canarias, Ed. Caja Insular de Ahorros (varios autores).

Nelken, M. (1921). La condición social de la mujer en España, Madrid, CVS Ediciones, Col. Ateneo. Edición de 1975.

Olivé Roig, S. (2013). Telégrafos. Un relato de su travesía centenaria, Ed. Ariel y Fundación Telefónica.

Pérez Jiménez, R. y Quintana Navarro, F. (2019). «Conectando el Atlántico: La radiotelegrafía en Canarias en el periodo de entreguerras», Anuario de Estudios Atlánticos, (65), 19.

Scanlon, G. (1976). La polémica feminista en la España contemporánea (1868.1974), Madrid, Edit. Siglo XXI, 1976.

Ueda, V. (2002). «¡Dígame! El trabajo de las telefonistas en las centrales telefónicas: un estudio comparado", Scripta Nova, vol. vi, n. ${ }^{\circ} 119$ (94), 1 de agosto de 2002.

\section{Fuentes documentales}

LACGCT: Libro de actas de la Comisión de Gobierno del Cabildo de Tenerife.

MPT: Fondos del Museo Postal y Telegráfico. 